\title{
Cancer gene therapy goes viral: viral vector platforms come of age
}

\author{
Urban Bezeljak \\ COBIK, Ajdovščina, Slovenia \\ Radiol Oncol 2022; 56(1): 1-13. \\ Received 25 November 2021 \\ Accepted 4 January 2022 \\ Correspondence to: Urban Bezeljak, Ph.D., COBIK, Mirce 21, 5270 Ajdovščina, Slovenia. E-mail: urban.bezeljak@cobik.si \\ Disclosure: No potential conflicts of interest were disclosed. \\ This is an open access article under the CC BY-NC-ND license (http://creativecommons.org/licenses/by-nc-nd/4.0/).
}

Background. Since the advent of viral vector gene therapy in 1990s, cancer treatment with viral vectors promised to revolutionize the field of oncology. Notably, viral vectors offer a unique combination of efficient gene delivery and engagement of the immune system for anti-tumour response. Despite the early potential, viral vector-based cancer treatments are only recently making a big impact, most prominently as gene delivery devices in approved CAR-T cell therapies, cancer vaccines and targeted oncolytic therapeutics. To reach this broad spectrum of applications, a number of challenges have been overcome - from our understanding of cancer biology to vector design, manufacture and engineering. Here, we take an overview of viral vector usage in cancer therapy and discuss the latest advancements. We also consider production platforms that enable mainstream adoption of viral vectors for cancer gene therapy.

Conclusions. Viral vectors offer numerous opportunities in cancer therapy. Recent advances in vector production platforms open new avenues in safe and efficient viral therapeutic strategies, streamlining the transition from lab bench to bedside. As viral vectors come of age, they could become a standard tool in the cancer treatment arsenal.

Key words: viral vector; gene therapy; oncolytic virus; immunotherapy; bioprocess platform

\section{Introduction}

Cancer remains a major public health concern worldwide and is the second leading cause of death in Europe and the United States, with one-intwo to one-in-three chance to develop an invasive cancer during individual's lifetime. ${ }^{1}$ In Slovenia, cancer caused over $35 \%$ deaths in males in 2016, which is the highest share in European Union. ${ }^{2}$ As a result of positive lifestyle changes and advances in tumour detection and treatment, we can observe a continuous drop in mortality rates in the last 20 years. ${ }^{3}$ Despite, current treatments like chemotherapy, surgery and radiation commonly have debilitating side effects. Consequently, new therapeutic options are becoming available to curb the tremendous death toll and increase the quality of life for cancer survivors. ${ }^{4}$ In this review we will focus on cancer therapeutics in form of viral gene therapy vectors and oncolytic viruses (OVs).

Viral vectors are an attractive drug delivery option due to their evolved efficiency to transduce human cells. Compared to other delivery methods, viruses are also easier to use in targeted transfer of genetic cargo. That is why modified viruses are used as reliable and safe gene therapy vectors in cancer and hereditary disease treatment. ${ }^{5}$ However, early attempts at viral gene therapy came too soon for the budding technology, which lead to controversy and poor public image. For example, the initial trials for severe combined immunodeficiency (SCID) saw only limited improvement and adenovirus vector-associated complications lead to tragic death of Jesse Gelsinger in 1999. ${ }^{6}$ The tides turned in later years, when viral vectors were successfully used as ex vivo hematopoietic gene delivery de- 
vices for severe $\beta$-thalassemia, SCID and WiskottAldrich syndrome. In 2003, Gendicine was the first approved adenoviral cancer gene therapeutic in China. It took almost another decade to see the first gene therapy approval in Europe, where the adeno-associated virus (AAV) alipogene tiparvovec (Glybera, uniQure) received authorisation for lipoprotein lipase deficiency (LPLD) treatment in 2012. Since then, many other therapies reached regulatory approval for in- or ex vivo gene delivery.7,8 More recently, novel vector-based vaccines are key in battling the coronavirus disease 2019 (COVID-19) pandemic on an unprecedented scale. ${ }^{9,10}$ This was made possible by the constant development of viral vector production and purification platforms, which had their roots in viral vector gene therapy. As the technology matures, the rapid turnaround of vector design and scalable particle production capacities hold promise to equally revolutionize cancer gene therapy. Indeed, over two thirds of gene therapy clinical trials are focused on cancer treatment, with many drug candidates in late development stages. ${ }^{11}$

Oncolytic therapy represents another use of viral vectors. It was sparked by serendipitous observations of transient remissions when cancer patients contracted viral infections. ${ }^{12}$ This led to experimentation with natural pathogens to help cure tumours, mostly with little success. Although initial attempts of viral oncolytic therapy were ineffective, the genetic engineering revolution enabled development of effective OVs in 1990s. ${ }^{13,14}$ Thirty years later, three oncolytic therapeutics are approved for use, with many more entering the clinics. Overall, oncolytic and viral gene delivery vectors have great potential to complement established (immuno)therapy approaches. These advanced nanotherapeutics are armed with a wide variety of genetic elements that take advantage of essential hallmarks of cancer, harnessing the accumulated knowledge in cancer biology, immunology and virology. Examples of therapeutic viral vector platforms, their opportunities and challenges are discussed below.

\section{Viral vectors}

At present, over 1000 clinical trials for cancer therapy with viral vectors are underway worldwide (Figure 1). ${ }^{11}$ The use of virus particles in cancer treatment can be broadly classified in two groups: as gene delivery vehicles and OVs. ${ }^{15,16}$ For gene delivery, lentiviral, adenoviral and AAV vector chas-

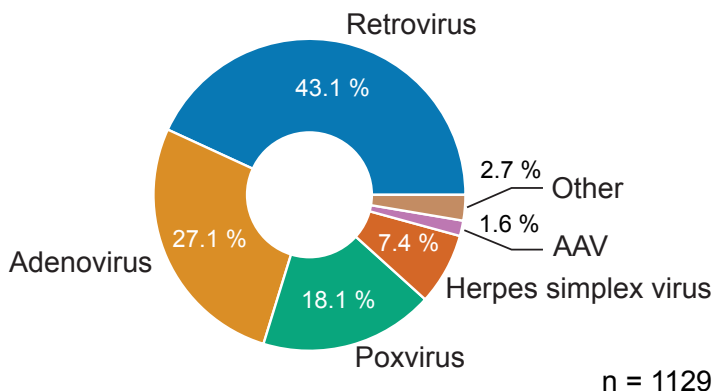

FIGURE 1. Use of viral vectors in clinical trials to treat cancer. Overall, retrovirus viral family vectors are the most widespread. These include lenti- and gammaretroviruses, which are used in adoptive cell therapy. Other popular vectors for cancer treatment are adenovirus, poxvirus like vaccinia, herpes simplex virus (HSV) and adeno-associated virus (AAV). Measles virus, vesicular stomatitis virus (VSV) and poliovirus are some of the other vectors that are not explicitly depicted. Data on all open cancer trials are from Wiley Journal of Gene Medicine Gene Therapy Clinical Trials Worldwide database (retrieved October 2021).11

sis are used - depending on the specific application and targeting specificity. OVs encompass many viral families and are often additionally armed to eradicate the tumour and induce anti-cancer immune response. ${ }^{4}$ The main difference between vectors in gene- and oncolytic therapy is their replicative potential. Gene therapy vectors are specifically engineered to prevent replication. Consequently, they function as nanoparticle drug delivery vehicles and cannot actively infect host cells. In contrast, OVs are less attenuated and can replicate in infected tissues. Each of these vector designs has its own set of advantages and disadvantages, which also depend on the clinical indication. Below, we overview some of the most widely used viral vectors for cancer treatment that were either approved for clinical use or introduce exciting new concepts for future therapies.

\section{Gene delivery}

Gene delivery vectors are used to transfer therapeutic genetic material to target tissues. In cancer therapy this includes tumour suppressor genes, tumour-associated antigens (TAAs), pro-inflammatory factors, immune checkpoint inhibitors, anti-angiogenic proteins, small interference RNA (siRNA), cancer stroma-degrading enzymes and cytotoxic convertases. ${ }^{17}$ In addition, vector gene delivery is used to reprogramme therapeutic cells $e x$ vivo for adoptive cell therapy like chimeric antigen receptor (CAR) $\mathrm{T}$ and natural killer (NK) cells. ${ }^{18,19}$ Here, we present the most well-known viral vec- 


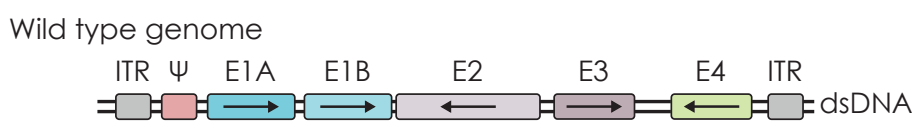

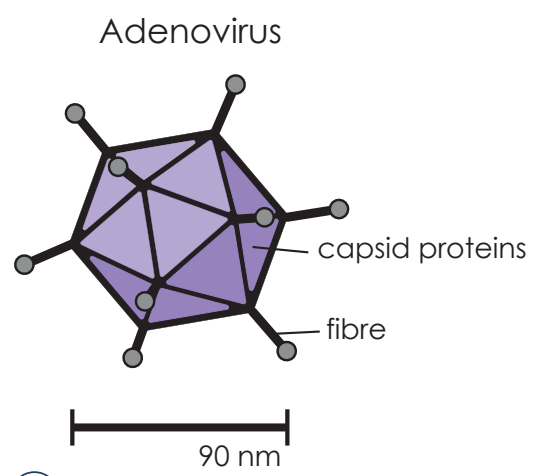

(A)

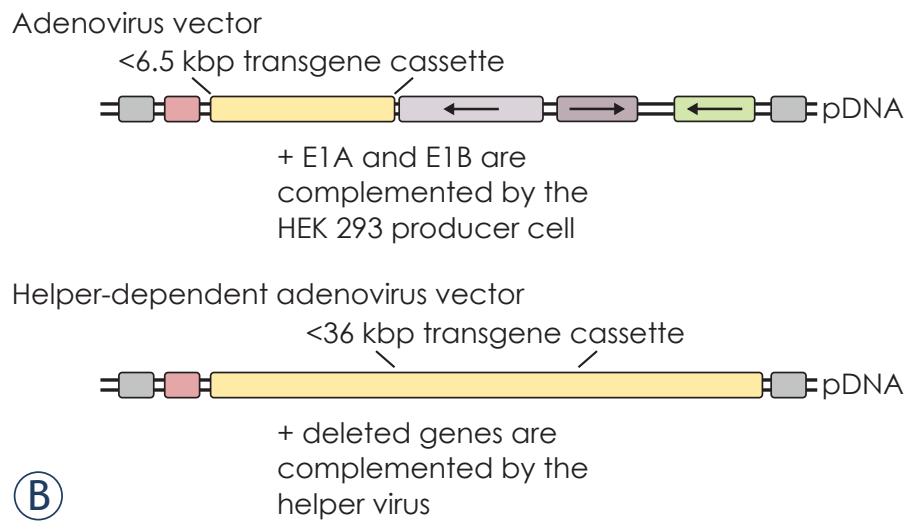

Adenovirus vector

$<6.5 \mathrm{kbp}$ transgene cassette

$+E I A$ and $E I B$ are

complemented by the

HEK 293 producer cell

complemented by the

helper virus

FIGURE 2. Overview of adenovirus vector design. (A) Schematic representation of adenovirus structure. Adenoviruses are nonenveloped $90 \mathrm{~nm}$ particles with pointing fibre rods. (B) Outline of wild type adenovirus genome, the first-generation adenovirus vector plasmid and helper-dependent adenoviral vector plasmid with the transgene expression cassette. The wild type genome highlights key early genes, while other genetic elements are omitted for clarity. The first-generation adenovirus vector particles are assembled in HEK 293 cell line by transgene vector plasmid transfection. Additionally, the helper-dependent vector assembly also requires infection with a helper virus.

ITR = inverted terminal repeat; $d \mathrm{~d} D N A=$ double-stranded DNA; pDNA = plasmid DNA.

tor platforms: adenovirus, which is used in in vivo gene therapy and retroviruses that are used for $e x$ vivo gene delivery. In addition, we discuss AAVs, which are currently the most exciting vector platform and will likely set trends in cancer gene therapy in the future.

\section{Adenovirus vectors}

Native adenoviruses are $90 \mathrm{~nm}$ icosahedral particles (Figure 2A) that commonly cause respiratory, gastrointestinal, urinary and keratoconjunctivitis infections in humans. Their ubiquity results in high proportion of life-long immunity in human population towards the most common serotypes. ${ }^{20}$ Adenoviruses have 36 kilobase pair (kbp) linear double-stranded DNA (dsDNA) genome consisting of over 30 genes that are flanked by inverted terminal repeats (ITRs) and a capsid-packing signal sequence $\psi$. The adenoviral genes are divided into early (E) and late (L) genes, depending on their expression pattern. Early expressed regulatory proteins interact with the host cell and initiate viral genome replication. On the other hand, late genes encode structural proteins that form the virion. ${ }^{21}$ Adenoviral vectors were developed by deleting key regulatory genes, which depend on the desired transgene size and application (Figure 2B). Replication-competent adenovirus vectors are used in oncolytic cancer therapy, while non-replicative deletion mutants are gene delivery vehicles. In the first generation of adenovirus vectors, the essential early E1A and E1B genes are replaced by constitutive expression cassette with transgene for gene delivery. Additionally, E2, E3 and E4 genes can also be deleted to accommodate larger therapeutic inserts of $10 \mathrm{kbp}$ and improve performance. For viral vector assembly, the modified adenovirus genome is expressed from plasmid DNA in human embryonic kidney (HEK) 293 cell line that complements for deleted E1, E2 and E4 genes. Lastly, as much as $36 \mathrm{kbp}$ inserts can be accommodated in helperdependent adenovirus vectors that retain only ITR and genome packaging sequence $\psi$, rest is filled with one or several transgene expression cassettes. All adenoviral proteins needed for vector replication, packaging and assembly are provided by the replication-competent helper virus, which has its packaging signal flanked by loxP recombination sites. The helper-dependent vector production takes place in cell lines expressing Cre recombinase that specifically excises the loxP-flanked helper $\psi$ sequence. This ensures only the transgene vector retains the $\psi$ packaging signal and is incorporated in the budding viral particles. Remaining helper virus contaminants are eliminated in the following chromatography purification process. Adenoviral vectors have broad tropism and do not integrate 
into target cell genome. Instead, the delivered genetic material remains episomal. ${ }^{15,22}$

In gene delivery, acquired and innate immunity towards adenovirus vectors is hindering their application. For example, the most widely used Ad5 serotype has 50\% seroprevalence in North America and over $90 \%$ in Côte d'Ivoire. ${ }^{15}$ Additionally, the adenoviral capsid and nucleic acid stimulates components of the complement system and Tolllike receptors (TLR). This raises safety concerns and efficacy issues for systemic adenoviral gene delivery in vivo. However, the intrinsic vector immunogenicity can also be harnessed in local cancer therapy by engaging the immune system and promoting anti-tumour responses. ${ }^{20}$

In 2003, the adenovirus-based Gendicine became the first registered cancer gene therapy treatment. Gendicine is an E1- and E3-deletion Ad5 viral vector, which encodes tumour suppressor p53 under Rous sarcoma virus (RSV) promoter regulation. The loss of p53 protective function is associated with at least half of cancers. ${ }^{23}$ Once the Ad5 vector delivers p53 transgene, it resumes anti-tumour function by promoting cancer cell apoptosis and stimulating the immune response. It received approval in China for advanced head and neck cancer treatment. ${ }^{23,24}$

Adenovirus vectors are also used to deliver cancer suicide genes that convert prodrugs to cytotoxic compounds. Examples include 5-fluorouridine (5-FU)-producing cytosine deaminase, purine nucleoside phosphorylase (PNP) that converts fludarabine phosphate (F-ara-AMP) to toxic 2-fluoroadenine, and ganciclovir-converting thymidine kinase (TK). ${ }^{15}$ Sitimagene ceradenovec (Cerepro, Ark Therapeutics) is a first-generation Ad5 vector that expresses convertase from herpes virus HSVTK. In 2005 it entered phase 3 trial for treatment of glioblastoma. In the trial, $1 \cdot 10^{12} \mathrm{Ad} 5$ vector particles were applied locally into the resected tumour and ganciclovir was administered intravenously. The study found no effect on survival, while the viral vector treatment improved time to re-intervention or death after resection - the primary trial endpoints. ${ }^{25}$ Despite this, the Cerepro marketing application in Europe was withdrawn in 2010. ${ }^{26}$

Similarly, immunostimulatory adenovirus cancer gene therapy was used to promote interferon alpha and beta (IFN $\alpha,-\beta)$, interleukin 2 (IL-2) and Fms-like tyrosine kinase 3 ligand (Flt3L) expression. ${ }^{20} \mathrm{~A}$ phase 3 trial for bacille CalmetteGuérin (BCG)-unresponsive bladder cancer with nadofaragene firadenovec, a replication deficient vector expressing IFN $\alpha$, recently reported favourable results. ${ }^{27}$ The non-muscle-invasive and BCG-unresponsive bladder cancer currently does not have efficient non-surgical treatments, which are often the only option for many patients. Adenoviral gene therapy is a promising alternative, since local administration led to 60- and 30\% complete response rate after 3 and 12 months, respectively. ${ }^{28}$ Lastly, the engineered chimpanzee ChAdOx1 vector vaccine platform - also used by the AZD1222 COVID-19 vaccine (Vaxzevria, Oxford-AstraZeneca) - is aimed at prostate cancer treatment in combination with checkpoint inhibitors. ${ }^{10,29,30}$ The cancer vaccine treatment consists of ChAdOx1 immunization against 5T4 tumour antigen and a Modified Vaccinia Ankara (MVA) vector boost. The phase 1 trial confirmed vaccine safety and immunogenicity, while phase $1 / 2$ efficacy trial was expected to complete in $2021 .{ }^{31}$ Overall, adenoviral gene delivery remains a promising venue for cancer therapy, either alone or in combination with radiotherapy, chemotherapy or checkpoint inhibitors. ${ }^{32}$ Also, the ease of industrial scale-up and established Good Manufacturing Practice (GMP) processes will further promote adenoviral platform for in vivo patient gene delivery. ${ }^{33}$

\section{Adeno-associated virus vectors}

Adeno-associated viruses (AAVs) hold great promise in the gene therapy field. AAVs do not cause any human disease, are non-replicative and have broad tissue tropism. AAVs are $25 \mathrm{~nm}$ icosahedral viruses (Figure $3 \mathrm{~A}$ ) with single-stranded DNA (ssDNA) genome, which naturally lacks many key regulatory genes for replication and expression. The missing genes are instead complemented with adenoviral co-infection of the host cell. Alternatively, herpes simplex and baculovirus can also provide the helper function. For gene delivery, the AAV genome is "gutted" - devoid of all viral genes - and replaced with transgene expression cassette (Figure 3B). The major AAV vector downside is its relatively low capacity for transgene inserts - it can accommodate $4.7 \mathrm{kbp}$ of genetic cargo, which can be limiting for many applications. ${ }^{15,34}$ The therapeutic AAV particles are commonly produced from three plasmid constructs in transfected HEK 293 cells, which already encode the adenoviral E1 helper gene. The vector plasmids contain the ITR-flanked transgene, AAV rep and cap genes and the adenoviral E2, E4 and VA genes, respectively. A more scalable solution is possible with Sf9 insect cells, which are co-infected with ITR-transgene and AAV cap/rep baculoviruses, respectively. ${ }^{35}$ 


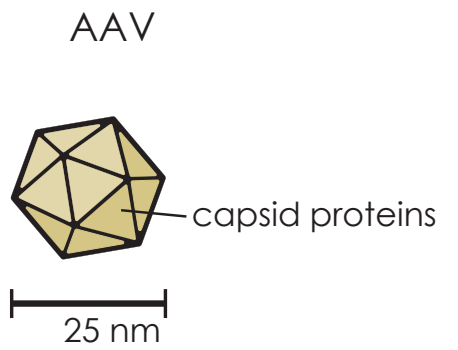

(A)
Wild type genome

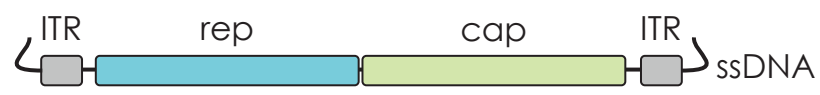

AAV vector

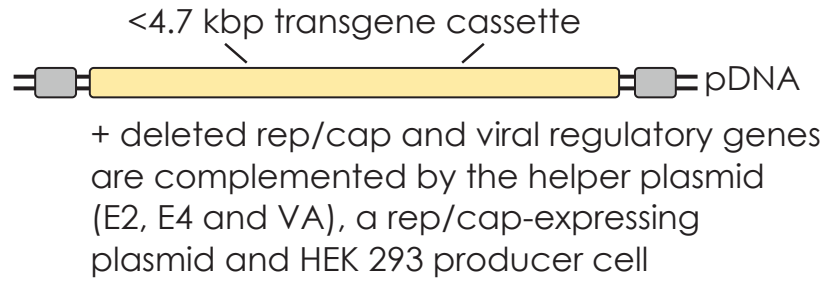

FIGURE 3. Overview of AAV vector design. (A) Schematic representation of AAV structure. AAV virions are non-enveloped 25 nm icosahedral particles. (B) Outline of wild type AAV genome and AAV vector plasmid with the transgene expression cassette. AAV vector particles are assembled in adenoviral El-expressing HEK 293 cell line, which is co-transfected with transgene AAV vector plasmid, a helper plasmid and a rep/cap plasmid. Alternatively, AAV vectors can be produced in insect cells, which are coinfected with ITR-flanked transgene and rep/cap recombinant baculoviruses.

ITR = inverted terminal repeat; pDNA = plasmid DNA; sSDNA = single-stranded DNA

Different AAV serotypes display distinct tropism, but they generally require AAV receptor (AAVR) expression, heparin sulphate peptidoglycans, sialic acid or galactose with several co-receptors for cell transduction. ${ }^{36}$ Once the vector enters the cell, it travels to nucleus and uncoats the transgene DNA, which persists as concatemerized episomal circle for many years..$^{35}$ Currently, AAV vectors are the most successful in treatment of monogenic diseases like spinal muscle atrophy (SMA) with onasemnogene abeparvovec (Zolgensma, Novartis). ${ }^{37}$ In contrast, AAV-based cancer therapies are still in early development. However, the modular vector design enables new promising approaches to targeted gene delivery. ${ }^{38}$

For instance, AAVs that cross the blood-brain barrier and are specific for central nervous system could be used for treatment of invasive glioblastoma. ${ }^{39}$ To improve cancer specificity, wild-type AAV capsids can also be engineered to target cell surface tumour antigens. ${ }^{40,41}$ For example, AAV2 was modified to bind HER2 receptor by inserting designed ankyrin repeat proteins (DARPins) into the AAV capsid. ${ }^{42}$ The researches later used these Her2-AAVs to specifically deliver checkpoint inhibitors against programmed cell death protein-1 (PD-1) and HSVTK suicide gene in a mice xenograft model. ${ }^{43,44} \mathrm{~A}$ single systemic injection of Her2-AAV vector, armed with HSV-TK, lead to considerable tumour mass reduction in combination with ganciclovir. ${ }^{44}$ On the other hand, PD-1 inhibition lead to only marginal tumour clearance in combination with chemotherapy. ${ }^{43}$ In an ex vivo application, an AAV6 vector was used to prepare allogenic CAR-T cells by replacing the endogenous $\mathrm{T}$ cell receptor (TCR) with CD19 CAR through targeted cleavage and homologous repair. ${ }^{45,46}$ In the future, AAVs could also be used for CAR-T cell generation in vivo, replacing the challenging retroviral $\mathrm{T}$ cell modification. This concept of "AAV delivering CAR gene therapy" (ACG) was proved on a T-cell leukaemia animal model, where murine immune cells were reprogrammed to express CD4 CAR. ${ }^{47}$ Finally, therapeutic AAVs are developed to include CRISPR/Cas gene editing components. This combination of powerful biotechnology platforms promises highly efficient tumour delivery and precise oncogene knock-out or silencing. ${ }^{48,49}$ To this end, a sub-4.7 kbp CRISPR/Cas13a that distinguishes between wild type and oncogenic KRAS G12D was constructed and tested in cell culture. A similar AAV vector with oncogene-specific Cas13a could someday induce tumour eradication through mRNA silencing. ${ }^{50}$

Overall, AAV particles are less immunogenic compared to other vector types, although majority of adults have pre-existing neutralizing antibodies that can affect AAV-based gene therapy efficency. ${ }^{51}$ Also, AAVs are regarded as very safe due to their non-toxic nature and expected lack of genome integration. However, a recent long-term study of AAV-treated dogs with haemophilia raised concerns as numerous vector integration events were surprisingly discovered in vicinity of cancer-associated genes. ${ }^{52}$ Nonetheless, the superior versatility makes AAVs currently the up-and-coming method for gene delivery in vivo..$^{53}$ 
Lentivirus

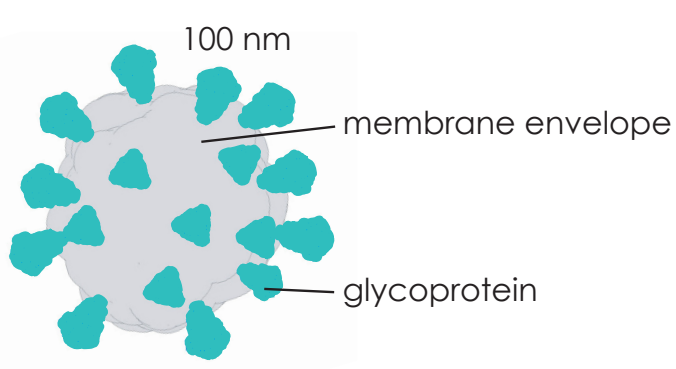

(A)
Wild type genome

LTR gag pol env rev LTR

Lentivirus vector

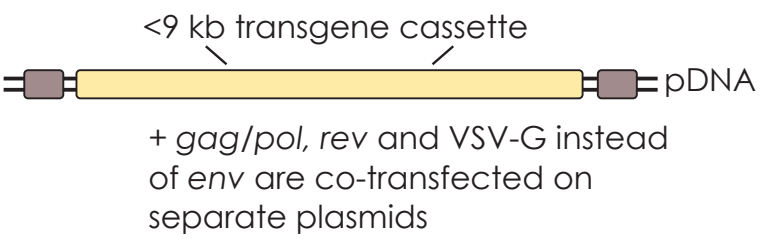

FIGURE 4. Overview of lentivirus vector design. (A) Schematic representation of lentivirus structure. Lentiviruses are 100 nm enveloped particles with exposed glycoprotein that defines the virus and vector tropism. ${ }^{55}$ (B) Outline of wild type lentivirus genome and lentivirus vector plasmid with the transgene expression cassette. Only key genetic elements are highlighted in the genome structure, rest are omitted for clarity. Lentivirus particles are assembled in mammalian cell culture by co-transfection of four plasmids: the transgene plasmid, gag/pol and rev packaging plasmids and VSV-G expression plasmid.

PDNA = plasmid DNA; sSRNA = single-stranded RNA; LTR= long terminal repeat

\section{Lenti- and gammaretrovirus vectors}

While adenovirus and AAV vectors are predominantly used to deliver gene drugs in vivo, retrovirus vectors like lenti- and gammaretroviruses are the most common choice for transformation of isolated patient cells ex vivo. Another distinction is their structure: retroviruses are enveloped $100 \mathrm{~nm}$ particles (Figure 4A), while adenoviruses and AAVs have smaller and more rigid proteinaceous shells. Lentivirus family include human- (HIV-1, HIV-2), simian- (SIV) and feline immunodeficiency viruses (FIV). In fact, the widely used lentiviral vectors are derived from HIV-1 that have been modified not to cause disease and to express vesicular stomatitis virus G glycoprotein (VSV-G) instead of the native envelope (env) protein. Pseudotyping the lentivirus particles with VSV-G increased the vector productivity, stability and infectivity, as well as broadened its tropism for different cell types and tissues. ${ }^{54-56}$ To guarantee vector safety, the majority of HIV-1 RNA genome is deleted. Only three key structural and regulatory genes remain: gag, pol and rev. Deletion of viral accessory proteins renders the lentiviral vector harmless. What is more, gag/pol, rev, VSV-G and the transgene $(<9 \mathrm{kbp})$ are divided on separate plasmids in the producing cell lines, preventing assembly of replication-competent virus through recombination (Figure 4B). ${ }^{57}$ This way only the transgene is included in the lentivirus particles, while other genetic elements remain behind. The produced lentivirus vectors cannot replicate and can transfer the therapeutic gene with high efficiency. ${ }^{58}$ The delivered transgene RNA sequence is flanked by modified long terminal repeats (LTR), promoter, packaging and reverse-transcription elements. This expression cassette is integrated into genome of transduced cells, ensuring long-term expression in dividing and non-dividing cells. Alternatively, non-integrating lentiviral vectors (NILVs) were developed that persist as episomal DNA. NILVs circumvent the safety concerns regarding oncogenic potential of integration mutagenesis and offer prolonged transgene expression. 59,60

In cancer therapy, lentiviral vectors are most used for ex vivo modification of $\mathrm{T}$ and NK cells. Particularly, CAR-T cells are successful in treating relapsed and refractory non-Hodgkin lymphoma and acute lymphoblastic leukaemia (ALL), resulting in the first FDA-approved therapy tisagenlecleucel (Kymriah, Novartis) in 2017.61,62 There, the patient $\mathrm{T}$ cells are harvested by leukapheresis, activated and transduced with lentivirus vector, which encodes CD19 CAR. The lentivirus is produced under biosafety level 2 (BSL-2) GMP conditions in transfected HEK 293T cells, purified and sterile filtered before $\mathrm{T}$ cell transduction. ${ }^{63,64} \mathrm{~A}$ dendritic cell (DC)-specific lentiviral cancer vaccine LV305 was also used to promote expression and immune presentation of New York Esophageal Squamous Cell Carcinoma-1 (NY-ESO-1) cancer antigen. The nonintegrating vector is pseudotyped with Sindbis virus envelope glycoprotein that binds CD209 receptor on DC. In the first-in-human phase 1 study, LV305 vaccination induced $\mathrm{CD}^{+}$and $\mathrm{CD} 8^{+} \mathrm{T}$ cell 
responses against NY-ESO-1-expressing tumours. Based on these results, further LV305 combination therapies are planned. ${ }^{65}$

Gammaretrovirus vectors, which are derived from murine leukaemia virus (MLV), are another type of retroviral vectors. In contrast to lentivirus, gammaretrovirus vector infects only actively dividing cells and is prone to integrate into gene regulatory regions, raising concerns for insertional oncogenesis. ${ }^{56}$ Nevertheless, axicabtagen ciloleucel (Yescarta, Gilead) is a CD19 CAR-T cell therapy for diffuse large B-cell lymphoma, which utilizes gammaretroviral vector for chimeric receptor gene delivery. ${ }^{66}$ Another gammaretroviral therapeutic is vocimagene amiretrorepvec (Toca 511), which encodes yeast cytosine deaminase that converts prodrug 5-fluorocytosine to toxic 5-FU in glioma cells. ${ }^{67}$ Despite promising results from mouse brain tumour models, a phase $2 / 3$ did not show improved patient survival compared to standard-of-care after Toca 511 injection. ${ }^{68}$ Lenti- and gammaretroviral vectors will remain the method of choice for ex vivo stable cell transduction. Their use in cancer therapy will focus on next-generation CAR-T and -NK cells with improved potency and solid tumour treatment. ${ }^{69}$

\section{Oncolytic viruses}

In contrast to viral vectors for gene therapy, engineered OVs are replication-competent and more closely resemble their natural counterparts. In fact, tumour regressions after natural viral infections have been reported since the end of 19th century. ${ }^{13}$ The OV therapy takes advantage of rapidly dividing cancer cells, which often lack antiviral defence mechanisms present in normal cells. For example, misregulation of interferon, Wnt, Ras/MAPK, p53 and $\mathrm{pRb}$ signalling pathways leaves cancer vulnerable for viral infection. ${ }^{70,71}$ Consequently, OVs preferentially replicate in cancerous cells, resulting in lysis, tumour eradication and immune system engagement. Besides the viral tumour debulking, the immunostimulatory effect is especially important in "cold" tumours with few infiltrating lymphocytes, inhibitory tumour microenvironment (TME) and impaired antigen presentation. There, viral antigens, danger-associated molecular patterns (DAMPs), TAAs and neoantigens are released to TME during infected tumour cell lysis. Released factors are then presented to the innate and adaptive immune system, acting as self-adjuvating in si$t u$ cancer vaccine. This leads to localized inflammation, recruiting immune cells into TME and mount- ing response towards distant metastatic lesions. ${ }^{16,72}$ The multifaceted oncolytic virotherapy is potentiated with immune checkpoint inhibitors or CAR-T cells, which is reflected in multiple combination therapy approaches. ${ }^{73-75}$ Similar to gene delivery vectors, OVs are often armed with transgenes that additionally modulate the TME or the immune system, including matrix-degrading enzymes, cytokines, checkpoint inhibitors, therapeutic anti-/ nanobodies and bispecific $\mathrm{T}$ cell engagers (BiTEs). ${ }^{17}$ The OV therapy can even be custom-made for each individual patient. Personalized therapeutic vectors can be designed by inserting patient-specific antigens directly into viral envelope or by encoding the neoantigen sequences for gene delivery. ${ }^{76,77}$ A broad range of oncolytic vectors are used for cancer therapy, representing different viral families. Below we mention some examples, which have been extensively tested and reached late stages of clinical trials.

Replication-competent adenovirus vectors were the first oncolytic viruses to reach clinical trials in 1998 with ONYX-015. ${ }^{78}$ This adenoviral vector harbours E1B-55K deletion, which attenuates viral replication in normal cells. While ONYX-015 presented a remarkable safety profile, it conferred only limited responses in combination with chemotherapy for many different cancers. ${ }^{22}$ In 2005, a similar vector H101 (Oncorine) became the first approved oncolytic virotherapy for late-stage nasopharyngeal carcinoma treatment in China. The objective response rate of Oncorine in combination with chemotherapy was $76 \%$ versus $59 \%$ for chemotherapy alone in phase 3 trial. ${ }^{79}$ Altogether, hundreds of patients received Oncorine, which was well tolerated and without adverse side effects. ${ }^{22}$

It took 10 years for another OV to be approved by the FDA and EMA in 2015. T-VEC or talimogene laherparepvec (Imlgyc, Amgen) is an oncolytic herpesvirus that expresses immunomodulatory granulocyte macrophage colony-stimulating factor (GM-CSF) for advanced melanoma treatment. ${ }^{80}$ Non-modified herpes simplex virus type 1 (HSV-1) is a neurotropic human pathogen with $152 \mathrm{kbp}$ dsDNA genome and about 90 genes. For cancer therapy with T-VEC vector, HSV-1 from a clinical isolate was modified with infected cell protein 34.5 (ICP34.5) deletions, preventing virus replication in neurons and other slowly replicating cells. Conversely, this deletion increases HSV-1 replication specificity for tumour cells, while another deletion in ICP47 increases viral and tumour antigen presentation. Lastly, T-VEC is armed with two copies of GM-CSF to further promote activa- 
tion of local antigen-presenting cells. ${ }^{72,81}$ The virotherapy proved effective in phase 3 trial where $16 \%$ of patients showed durable response compared to $2 \%$ for GM-CSF treatment. The overall response rate was $26 \%$ for T-VEC and $6 \%$ for GMCSF alone. Furthermore, $64 \%$ of injected lesions more than halved in size, together with $34 \%$ and $15 \%$ of distal uninjected regional and visceral lesions, respectively. ${ }^{82}$ A similar antitumour T-VEC activity was recently reported for primary cutaneous B cell lymphoma. Interestingly, this phase 1 study also showed therapeutic virus replication in non-malignant cells and determined that induced immunological responses are more important in cancer eradication than selective viral cell lysis. ${ }^{83,84}$ Currently, combination therapy studies with T-VEC are underway for different cancers. ${ }^{16,85,86} \mathrm{~A}$ similar set of HSV-1 attenuations is also present in G47 $\Delta$ or teserpaturev (Delytact, Daiichi Sankyo), which is derived from a different parental strain. In 2021, it received conditional authorization for malignant glioma therapy in Japan. ${ }^{87}$ In contrast to T-VEC, which is armed with GM-CSF, Delytact does not encode any transgenes. ${ }^{88}$

The GM-CSF cytokine sequence is also loaded in oncolytic vaccinia virus pexastimogene devacirepvec (JX-594, Pexa-Vec) to promote in situ vaccine activity. Pexa-Vec is a TK-deleted poxvirus that demonstrated specificity for tumour cells, which often have increased TK levels that compensate the OV attenuation. In contrast to T-VEC, Pexa-Vec is administered systemically by intravenous injection for hepatocellular carcinoma (HCC), renal cell cancer and colorectal cancer treatment. ${ }^{74,89}$ In early HCC trials, Pexa-Vec replication was detected in cancer and tumour-associated endothelial cells, triggering specific immune response and destruction of tumour blood vessels. ${ }^{90,91}$ However, a phase 3 HCC trial with Pexa-Vec and a protein kinase inhibitor sorafenib was prematurely stopped due to lack of interim efficacy. ${ }^{92}$ A similar fate faced Prostvac-VF, a vaccinia and fowlpox prime-boost vector combination that delivers prostate cancerspecific antigen PSA and three additional immunostimulatory factors. The dual vector combination prevents antibody neutralization of the replicating viral particles, which resulted in increased survival for prostate cancer patients in phase 2 study. In contrast, a multicentre phase 3 trial did not confirm these findings, ending the study prematurely. Prostvac-VF combination therapies are still evaluated in phase 1 and 2 clinical trials. ${ }^{93-95}$

The complexity of viral vector genomes still poses a potential risk of recombination and acquired pathogenicity during vector production and therapy. Indeed, with an increasing number of effective OVs reaching late clinical trials and regulatory approvals, a particular care is given to biosafety monitoring and interaction of replication-competent vectors with the host and environment. Based on gathered experience, engineered oncolytic viral vectors remain a safe and promising venue for cancer treatment. The most common reported side effects are mild flu-like symptoms, while there was no documented uncontrolled transmission of the oncolytic virus. ${ }^{14}$ It is becoming clear that OVs offer unique benefits in tumour immunotherapy, particularly in combination with advanced cell therapies, chemotherapy and checkpoint inhibitors. ${ }^{96}$

\section{Production platforms}

Reliable production platforms are key to successful translation of viral vector therapies into clinics. Indeed, the development of robust manufacturing capabilities enabled the widespread adoption of recombinant biotherapeutics like monoclonal antibodies. Compared to proteins, viral vectors are orders of magnitude more complex, where minute changes between serotypes can affect production and purification strategies. What is more, conventional protein purification methods are often not appropriate for shear-sensitive viral particle isolation. Enveloped vectors are easily ruptured during the purification process, which decreases the ratio of functional infectious particles in the final product. Consequently, it is no surprise that viral vector production platforms are continuously being optimized and are yet to reach their full potential on the industrial scale. ${ }^{6,97}$ Moreover, viral vectors for gene delivery and oncolysis span several viral families and are further genetically engineered to ensure safety and anti-tumoral potencies, adding to the diversity. This offers exciting opportunities for tailored therapies, but also raises challenges in manufacturing and regulation. Thus, paths to viral vector platform success and adoption are specific for each therapeutic.

In order to support consistent production and reliable scale-up to clinical-grade drug manufacture, an extra care has to be taken in initial selection of viral vector production platforms. Established mammalian cell cultures are most widely used for vector propagation. This makes sense since therapeutic viral vectors originate from natural viruses that co-evolved with vertebrate hosts. The producer cell lines include African green monkey 
Vero cells, human HEK 293 and HeLa cells and baby hamster kidney (BHK) line. ${ }^{98}$ They are handled under BSL-2 regulations to limit vector dissemination and contamination with adventitious agents. ${ }^{99}$ Mammalian cell lines are primarily adherent - growing attached to a solid support. For production, adherent cells are grown on microcarriers in bioreactors, on multilayer tissue plates and roller bottles. However, suspension cell cultures are preferred for easier scale-up. Luckily, many adherent cell lines were successfully adapted for suspension growth in stirred-tank and rocking Wave bioreactors. ${ }^{97,98,100}$ For example, the adenovirustransformed HEK 293 cells are easily adapted for suspension culture and are the most widely used cell line for adenoviral and AAV vector production.

Vector components and helper constructs are delivered to the selected producer cells with transient transfection or infection, which represent a bottleneck and a significant expense in viral vector production for clinical trials. ${ }^{101}$ In principle, stable cell lines overcome transfection issues in large scale production. However, assembled vector components are often toxic for the host cell. ${ }^{5}$ This is why stable lentiviral production lines rely on inducible vector expression using TetON/OFF system. ${ }^{102}$ Alternatively, the cytotoxic VSV-G envelope protein and HIV-1 protease can be swapped with Ampho MLV 4070A glycoprotein and T26S modified protease to generate stable lentiviral vector producers LentiPro26 from HEK 293T cells. ${ }^{103}$ Besides mammalian cell cultures, insect cells are also increasingly used for vector assembly. Most commonly, Sf9 or HighFive ${ }^{\mathrm{TM}}$ insect cell lines are grown in suspension where therapeutic vector backbones are introduced through infection with recombinant baculoviruses. Insect cell expression system is fast to implement, scalable and has superior safety profile since insects are poor hosts for human pathogens. So far, insect cells are used to produce helper-free AAV vectors for gene therapy. ${ }^{104,105}$ Like with mammalian viral vector systems, insect cells are grown in single-use cell culture flasks and bioreactors to ensure reproducibility and sterility during manufacture for clinical use. ${ }^{101,106}$

Viral vector purification platforms encompass purification steps to generate highly pure vector particles that comply with stringent quality, safety and efficacy standards. Historically, purification relied on ultracentrifugation to separate the large viral particles from smaller producer cell contaminants. Nonetheless, this approach is not scalable and does not guarantee elimination of biophysi- cally similar particles. ${ }^{107}$ Instead, chromatographybased purification processes are taking the centre stage. ${ }^{108}$ Due to diversity of viral vectors - ranging from small $25 \mathrm{~nm}$ AAV particles to large enveloped vaccinia vector, which exceeds $300 \mathrm{~nm}$ in size - a universal purification process does not exist and has to optimized for each application. Generally, the purification process starts with vector particle harvesting, where secreted vectors like lentiviruses, herpes and poxviruses are separated from cellular debris with centrifugation or filtration, while intracellular adenoviral and AAV particles often require cell lysis for release. Then, the collected harvest is extensively purified over a series of different chromatography columns and tangentialflow filtration (TFF) cassettes to obtain pure therapeutic vector particles. In the end, the purified cancer drug is exchanged to the final formulation solution and sterile filtered through $0.2 \mu \mathrm{m}$ pores, which can be problematic for some larger vectors. Instead, the entire production can be operated under controlled sterile conditions. ${ }^{97,108}$ The final dose of vector particles varies from $10^{9}$ for ex vivo gene delivery up to $10^{14} / \mathrm{kg}$ for AAV-based gene therapy. ${ }^{108,109}$ However, all purification steps are associated with loss of infectious particles, which increases the cost of manufacture. The complexity of viral vector production and low yields result in exceedingly high price of advanced therapeutics. For example, CAR-T therapies Kymriah and Yescarta cost $\$ 373,000$, while Zolgensma gene therapy was marketed at \$2.125 million at launch in 2019.15,110 The constant improvements in production technology will make the viral vector therapeutics more accessible to the patients. ${ }^{111}$

With many viral vector therapeutics reaching final clinical stage and regulatory approvals, the attention is focused on vector particle production platforms that support scalable industrial scale production. This is essential to bring down the cost of therapies, which is often prohibitive. New biotechnological solutions like gene editing, bioreactor cultivation and multimodal chromatography are boosting cell-based productivity and improving particle purity. Novel analytical methods are also improving the quality monitoring of the final product. In gene therapy, great emphasis is given to ensuring a high ratio of functional infectious vector particles versus defective and empty capsid contaminants. ${ }^{112}$ These exciting developments are enabling the viral vector platforms to produce safe and potent drugs to combat cancer - as monotherapies or in combination with other therapeutic venues. 


\section{Conclusions}

Viral vectors represent $100 \%$ of approved gene therapeutics and over $60 \%$ of delivery devices in gene therapy trials, including oncolytic virotherapy. ${ }^{11}$ This accumulation of knowledge helps us identify where vector particles can provide the most benefits. Based on recent success and rapid advances in the field, the clinical viral vector use will continue to increase. Particularly, combination therapy with complementary radiotherapy, chemotherapy and immunotherapies like CAR-T and checkpoint inhibitors hold great promise. Due to modular vector genome design, novel biotherapeutics like BiTEs, cytokines and CRISPR/Cas can be encoded in the genetic cargo to expand the repertoire of anti-tumour potency. ${ }^{113}$ In addition to new vector development, approved viral drugs like T-VEC are being tested to treat several other solid tumours beyond melanoma. Finally, oncolytic viruses and non-replicative vectors can be used in prime-boost cancer vaccine regimens, covering the full spectrum of the discussed vector platforms. ${ }^{114}$

With obvious benefits to the viral vector oncotherapy, these engineered nanotherapeutics will continue to expand the cancer treatment repertoire. It seems like viral vector platforms are finally living up the high expectations thanks to the advances in biopharmaceutical manufacturing and our understanding of cancer and viral biology. In the future, many more vector particles will enter the clinics. Excitingly, their adaptable design will enable de novo engineering and repurposing of existing chassis for novel therapeutic approaches.

\section{Acknowledgments}

The author would like to thank A. Smole, R. Hudej and M. Peterka for valuable feedback on the manuscript.

\section{References}

1. Siegel RL, Miller KD, Fuchs HE, Jemal A. Cancer statistics, 2021. CA Cancer Clin 2021; 71: 7-33. doi: 10.3322/caac. 21654

2. Eurostat. Cancer statistics - statistics explained. [cited 2021 Nov 18] Available at: https://ec.europa.eu/eurostat/statistics-explained/index. php?title=Cancer statistics\#Deaths from cancer

3. Zadnik V, Zagar T, Lokar K, Tomsic S, Konjevic AD, Zakotnik B. Trends in population-based cancer survival in Slovenia. Radiol Oncol 2021; 55: 42-9. doi: 10.2478/raon-2021-0003

4. Cross D, Burmester JK. Gene therapy for cancer treatment: past, present and future. Clin Med Res 2006; 4: 218-27. doi: 10.3121/cmr.4.3.218
5. Goswami R, Subramanian G, Silayeva L, Newkirk I, Doctor D, Chawla K, et al. Gene therapy leaves a vicious cycle. Front Oncol 2019; 9: 1-25. doi: 10.3389/fonc.2019.00297

6. Sheridan C. Gene therapy finds its niche. Nat Biotechnol 2011; 29: 121-8. doi: $10.1038 /$ nbt.1769

7. Kotterman MA, Chalberg TW, Schaffer DV. Viral vectors for gene therapy: translational and clinical outlook. Annu Rev Biomed Eng 2015; 17: 63-89. doi: 10.1146/annurev-bioeng-071813-104938

8. Daley J. Gene therapy arrives. Nature 2019; 576: S12-3. doi: 10.1038/ d41586-019-03716-9

9. Sadoff J, Gray G, Vandebosch A, Cárdenas V, Shukarev G, Grinsztejn B, et al. Safety and efficacy of single-dose Ad26.COV2.S vaccine against Covid-19. N Engl J Med 2021; 384: 2187-201. doi: 10.1056/NEJMoa2101544

10. Voysey M, Clemens SAC, Madhi SA, Weckx LY, Folegatti PM, Aley PK, et al. Safety and efficacy of the ChAdOx1 nCoV-19 vaccine (AZD1222) against SARS-CoV-2: an interim analysis of four randomised controlled trials in Brazil, South Africa, and the UK. Lancet 2021; 397: 99-111. doi: 10.1016/ S0140-6736(20)32661-1

11. Ginn SL, Amaya AK, Alexander IE, Edelstein M, Abedi MR. Gene therapy clinical trials worldwide to 2017: an update. J Gene Med 2018; 20: 1-16. doi: 10.1002/jgm.3015

12. Dock $\mathrm{G}$. The influence of compliting disease upon leukaemia. Am J Med SCi 1904; 127: 563-92.

13. Kelly E, Russell SJ. History of oncolytic viruses: genesis to genetic engineering. Mol Ther 2007; 15: 651-9. doi: 10.1038/sj.mt.6300108

14. Miest TS, Cattaneo R. New viruses for cancer therapy: meeting clinical needs. Nat Rev Microbiol 2014; 12: 23-34. doi: 10.1038/nrmicro3140

15. Bulcha JT, Wang Y, Ma H, Tai PWL, Gao G. Viral vector platforms within the gene therapy landscape. Signal Transduct Target Ther 2021; 6: 53. doi: $10.1038 / \mathrm{s} 41392-021-00487-6$

16. Saxena M, van der Burg SH, Melief CJM, Bhardwaj N. Therapeutic cancer vaccines. Nat Rev Cancer 2021; 21: 360-78. doi: 10.1038/s41568-02100346-0

17. Wan PKT, Ryan AJ, Seymour LW. Beyond cancer cells: targeting the tumor microenvironment with gene therapy and armed oncolytic virus. Mol Ther 2021; 29: 1668-82. doi: 10.1016/j.ymthe.2021.04.015

18. Barrett DM, Singh N, Porter DL, Grupp SA, June CH. Chimeric antigen receptor therapy for cancer. Annu Rev Med 2014; 65: 333-47. doi: 10.1146/ annurev-med-060512-150254

19. Myers JA, Miller JS. Exploring the NK cell platform for cancer immunotherapy. Nat Rev Clin Oncol 2021; 18: 85-100. doi: 10.1038/s41571-020-0426-7

20. Shaw AR, Suzuki M. Immunology of adenoviral vectors in cancer therapy. Mol Ther Methods Clin Dev 2019; 15: 418-29. doi: 10.1016/j. omtm.2019.11.001

21. McConnell MJ, Imperiale MJ. Biology of adenovirus and its use as a vector for gene therapy. Hum Gene Ther 2004; 15: 1022-33. doi: 10.1089/ hum.2004.15.1022

22. Wold WSM, Toth K. Adenovirus vectors for gene therapy, vaccination and cancer gene therapy. Curr Gene Ther 2013; 13: 421-33. doi: 10.2174/1566 523213666131125095046

23. Peng Z. Current status of gendicine in China: recombinant human Ad-p53 agent for treatment of cancers. Hum Gene Ther 2005; 16: 1016-27. doi: 10.1089/hum.2005.16.1016

24. Wang $D$, Wang K, Cai Y. An overview of development in gene therapeutics in China. Gene Ther 2020; 27: 338-48. doi: 10.1038/s41434-020-0163-7

25. Westphal M, Ylä-Herttuala S, Martin J, Warnke P, Menei P, Eckland D, et al. Adenovirus-mediated gene therapy with sitimagene ceradenovec followed by intravenous ganciclovir for patients with operable high-grade glioma (ASPECT): a randomised, open-label, phase 3 trial. Lancet Oncol 2013; 14 823-33. doi: 10.1016/S1470-2045(13)70274-2

26. European Medicines Agency. Ark Therapeutics Ltd withdraws its marketing authorisation application for Cerepro (sitimagene ceradenovec). [cited 2021 Dec 21]. Available at: https://www.ema.europa.eu/en/news/ ark-therapeutics-Itd-withdraws-its-marketing-authorisation-applicationcerepro-sitimagene 
27. Kulkarni GS. Nadofaragene firadenovec: a new gold standard for BCGunresponsive bladder cancer? Lancet Oncol 2021; 22: 8-9. doi: 10.1016/ S1470-2045(20)30586-6

28. Boorjian SA, Alemozaffar M, Konety BR, Shore ND, Gomella LG, Kamat $A M$, et al. Intravesical nadofaragene firadenovec gene therapy for BCGunresponsive non-muscle-invasive bladder cancer: a single-arm, open-label, repeat-dose clinical trial. Lancet Oncol 2021; 22: 107-17. doi: 10.1016/ S1470-2045(20)30540-4

29. Dicks MD, Spencer AJ, Edwards NJ, Wadell G, Bojang K, Gilbert SC, et al. A novel chimpanzee adenovirus vector with low human seroprevalence: improved systems for vector derivation and comparative immunogenicity. PLoS One 2012; 7: e40385. doi: 10.1371/journal.pone.0040385

30. Mercuri E, Muntoni F, Baranello G, Masson R, Boespflug-Tanguy O, Bruno $C$, et al. Onasemnogene abeparvovec gene therapy for symptomatic infantile-onset spinal muscular atrophy type 1 (STR1VE-EU): an open-label, single-arm, multicentre, phase 3 trial. Lancet Neurol 2021; 20: 832-41. doi: 10.1016/S1474-4422(21)00251-9

31. Cappuccini F, Bryant R, Pollock E, Carter L, Verrill C, Hollidge J, et al. Safety and immunogenicity of novel 5T4 viral vectored vaccination regimens in early stage prostate cancer: a phase I clinical trial. J Immunother Cancer 2020; 8: 1-13. doi: 10.1136/jitc-2020-000928

32. Sato-Dahlman M, LaRocca CJ, Yanagiba C, Yamamoto M. Adenovirus and immunotherapy: advancing cancer treatment by combination. Cancers 2020; 12: 1295. doi: 10.3390/cancers 12051295

33. Lee CS, Bishop ES, Zhang R, Yu X, Farina EM, Yan S, et al. Adenovirusmediated gene delivery: potential applications for gene and cell-based therapies in the new era of personalized medicine. Genes Dis 2017; 4: 43-63. doi: 10.1016/j.gendis.2017.04.001

34. Xiao X, Li J, Samulski RJ. Production of high-titer recombinant adenoassociated virus vectors in the absence of helper adenovirus. J Virol 1998; 72: 2224-32. doi: 10.1128/JVI.72.3.2224-2232.1998

35. Samulski RJ, Muzyczka N. AAV-mediated gene therapy for research and therapeutic purposes. Annu Rev Virol 2014; 1: 427-51. doi: 10.1146/ annurev-virology-031413-085355

36. Srivastava A. In vivo tissue-tropism of adeno-associated viral vectors. Curr Opin Virol 2016; 21: 75-80. doi: 10.1016/j.coviro.2016.08.003

37. Mercuri E, Muntoni F, Baranello G, Masson R, Boespflug-Tanguy O, Bruno $C$, et al. Onasemnogene abeparvovec gene therapy for symptomatic infantile-onset spinal muscular atrophy type 1 (STR1VE-EU): an open-label, single-arm, multicentre, phase 3 trial. Lancet Neurol 2021; 20: 832-41. doi: 10.1016/S1474-4422(21)00251-9

38. Challis RC, Ravindra Kumar S, Chan KY, Challis C, Beadle K, Jang MJ, et al. Systemic AAV vectors for widespread and targeted gene delivery in rodents. Nat Protoc 2019; 14: 379-414. doi: 10.1038/s41596-018-0097-3

39. Xu X, Chen W, Zhu W, Chen J, Ma B, Ding J, et al. Adeno-associated virus (AAV)-based gene therapy for glioblastoma. Cancer Cell Int 2021; 21: 1-10. doi: 10.1186/s12935-021-01776-4

40. Santiago-Ortiz JL, Schaffer DV. Adeno-associated virus (AAV) vectors in cancer gene therapy. J Control Release 2016; 240: 287-301. doi: 10.1016/j. jconrel.2016.01.001

41. Hacker UT, Bentler M, Kaniowska D, Morgan M, Büning H. Towards clinical implementation of adeno-associated virus (AAV) vectors for cancer gene therapy: current status and future perspectives. Cancers 2020; 12: 1-30. doi: $10.3390 /$ cancers 12071889

42. Münch RC, Janicki $H$, Völker $I$, Rasbach $A$, Hallek $M$, Büning $H$, et al. Displaying high-affinity ligands on adeno-associated viral vectors enables tumor cell-specific and safe gene transfer. Mol Ther 2013; 21: 109-18. doi: 10.1038/mt.2012.186

43. Reul J, Frisch J, Engeland CE, Thalheimer FB, Hartmann J, Ungerechts G, et al. Tumor-specific delivery of immune checkpoint inhibitors by engineered AAV vectors. Front Oncol 2019; 9: 52. doi: 10.3389/fonc.2019.00052

44. Münch RC, Muth A, Muik A, Friedel T, Schmatz J, Dreier B, et al. Off-targetfree gene delivery by affinity-purified receptor-targeted viral vectors. Nat Commun 2015; 6: 6246. doi: 10.1038/ncomms7246

45. MacLeod DT, Antony J, Martin AJ, Moser RJ, Hekele A, Wetzel KJ, et al. Integration of a CD19 CAR into the TCR alpha chain locus streamlines production of allogeneic gene-edited CAR T cells. Mol Ther 2017; 25: 949-61. doi: 10.1016/j.ymthe.2017.02.005
46. Eyquem J, Mansilla-Soto J, Giavridis T, van der Stegen SJC, Hamieh M, Cunanan KM, et al. Targeting a CAR to the TRAC locus with CRISPR/Cas9 enhances tumour rejection. Nature 2017; 543: 113-7. doi: 10.1038/nature21405

47. Nawaz W, Huang B, Xu S, Li Y, Zhu L, Yiqiao H, et al. AAV-mediated in vivo CAR gene therapy for targeting human T-cell leukemia. Blood Cancer J 2021; 11: 119. doi: 10.1038/s41408-021-00508-1

48. Wang D, Zhang F, Gao G. CRISPR-based therapeutic genome editing: strategies and in vivo delivery by AAV vectors. Cell 2020; 181: 136-50. doi: 10.1016/j.cell.2020.03.023

49. Ibraheim R, Tai PWL, Mir A, Javeed N, Wang J, Rodríguez TC, et al. Selfinactivating, all-in-one AAV vectors for precision Cas9 genome editing via homology-directed repair in vivo. Nat Commun 2021; 12: 6267. doi: 10.1038/s41467-021-26518-y

50. Zhao X, Liu L, Lang J, Cheng K, Wang Y, Li X, et al. A CRISPR-Cas13a system for efficient and specific therapeutic targeting of mutant KRAS for pancreatic cancer treatment. Cancer Lett 2018; 431: 171-81. doi: 10.1016/j. canlet.2018.05.042

51. Calcedo R, Vandenberghe LH, Gao G, Lin J, Wilson JM. Worldwide epidemiology of neutralizing antibodies to adeno-associated viruses. J Infect Dis 2009; 199: 381-90. doi: 10.1086/595830

52. Venditti CP. Safety questions for AAV gene therapy. Nat Biotechnol 2021; 39: 24-6. doi: 10.1038/s41587-020-00756-9

53. Shirley JL, de Jong YP, Terhorst C, Herzog RW. Immune responses to viral gene therapy vectors. Mol Ther 2020; 28: 709-22. doi: 10.1016/j. ymthe.2020.01.001

54. Naldini L, Blömer U, Gallay P, Ory D, Mulligan R, Gage FH, et al. In vivo gene delivery and stable transduction of nondividing cells by a lentiviral vector. Science 1996; 272: 263-7. doi: 10.1126/science.272.5259.263

55. Cockrell AS, Kafri T. Gene delivery by lentivirus vectors. Mol Biotechnol 2007; 36: 184-204. doi: 10.1007/s12033-007-0010-8

56. Milone MC, O’Doherty U. Clinical use of lentiviral vectors. Leukemia 2018; 32: 1529-41. doi: 10.1038/s41375-018-0106-0

57. Dull T, Zufferey R, Kelly M, Mandel RJ, Nguyen M, Trono D, et al. A thirdgeneration lentivirus vector with a conditional packaging system. J Virol 1998; 72: 8463-71. doi: 10.1128/jvi.72.11.8463-8471.1998

58. Trono D. Lentiviral vectors: turning a deadly foe into a therapeutic agent. Gene Ther 2000; 7: 20-3. doi: 10.1038/sj.gt.3301105

59. Yáñez-Muñoz RJ, Balaggan KS, MacNeil A, Howe SJ, Schmidt M, Smith AJ, et al. Effective gene therapy with nonintegrating lentiviral vectors. Nat Med 2006; 12: 348-53. doi: 10.1038/nm1365

60. Philippe S, Sarkis C, Barkats M, Mammeri H, Ladroue C, Petit C, et al. Lentiviral vectors with a defective integrase allow efficient and sustained transgene expression in vitro and vivo. Proc Natl Acad Sci U S A 2006; 103 17684-9. doi: 10.1073/pnas.0606197103

61. Brentjens RJ, Davila ML, Riviere I, Park J, Wang X, Cowell LG, et al. CD19targeted T cells rapidly induce molecular remissions in adults with chemotherapy-refractory acute lymphoblastic leukemia. Sci Transl Med 2013; 5: 177ra38. doi: 10.1126/scitransImed.3005930

62. Vairy S, Garcia JL, Teira P, Bittencourt H. CTL019 (Tisagenlecleucel): CAR-T therapy for relapsed and refractory B-cell acute lymphoblastic leukemia. Drug Des Devel Ther 2018; 12: 3885-98. doi: 10.2147/DDDT.S138765

63. Levine BL, Miskin J, Wonnacott K, Keir C. Global manufacturing of CAR T cell therapy. Mol Ther Methods Clin Dev 2017; 4: 92-101. doi: 10.1016/j. omtm.2016.12.006

64. Levine BL. Performance-enhancing drugs: design and production of redirected chimeric antigen receptor (CAR) T cells. Cancer Gene Ther 2015; 22: 79-84. doi: 10.1038/cgt.2015.5

65. Somaiah N, Block MS, Kim JW, Shapiro GI, Do KT, Hwu P, et al. First-in-class, first-in-human study evaluating LV305, a dendritic-cell tropic lentiviral vector, in sarcoma and other solid tumors expressing NY-ESO-1. Clin Cancer Res 2019; 25: 5808-17. doi: 10.1158/1078-0432.CCR-19-1025

66. Kochenderfer JN, Feldman SA, Zhao Y, Xu H, Black MA, Morgan RA et al. Construction and preclinical evaluation of an anti-CD19 chimeric antigen receptor. J Immunother 2009; 32: 689-702. doi: 10.1097/ CJl.0b013e3181ac6138 
67. Ostertag D, Amundson KK, Lopez Espinoza F, Martin B, Buckley T, Galvão da Silva AP, et al. Brain tumor eradication and prolonged survival from intratumoral conversion of 5-fluorocytosine to 5-fluorouracil using a nonlytic retroviral replicating vector. Neuro Oncol 2012; 14: 145-59. doi: 10.1093/ neuonc/nor199

68. Cloughesy TF, Petrecca K, Walbert T, Butowski N, Salacz M, Perry J, et al. Effect of vocimagene amiretrorepvec in combination with Flucytosine vs standard of care on survival following tumor resection in patients with recurrent high-grade glioma: a randomized clinical trial. JAMA Oncol 2020; 6: 1939-46. doi: 10.1001/jamaoncol.2020.3161

69. Albinger N, Hartmann J, Ullrich E. Current status and perspective of CAR-T and CAR-NK cell therapy trials in Germany. Gene Ther 2021; 28: 513-27. doi: 10.1038/s41434-021-00246-w

70. Pikor LA, Bell JC, Diallo JS. Oncolytic viruses: Exploiting cancer's dea with the devil. Trends in Cancer 2015; 1: 266-77. doi: 10.1016/j.trecan.2015.10.004

71. Twumasi-Boateng K, Pettigrew JL, Kwok YYE, Bell JC, Nelson BH. Oncolytic viruses as engineering platforms for combination immunotherapy. Nat Rev Cancer 2018; 18: 419-32. doi: 10.1038/s41568-018-0009-4

72. Russell SJ, Peng KW. Oncolytic virotherapy: a contest between apples and oranges. Mol Ther 2017; 25: 1107-16. doi: 10.1016/j.ymthe.2017.03.026

73. Harrington K, Freeman DJ, Kelly B, Harper J, Soria JC. Optimizing oncolytic virotherapy in cancer treatment. Nat Rev Drug Discov 2019; 18: 689-706. doi: 10.1038/s41573-019-0029-0

74. Lawler SE, Speranza MC, Cho CF, Chiocca EA. Oncolytic viruses in cance treatment: a review. JAMA Oncol 2017; 3: 841-9. doi: 10.1001/jamaoncol.2016.2064

75. Lichty BD, Breitbach CJ, Stojdl DF, Bell JC. Going viral with cancer immunotherapy. Nat Rev Cancer 2014: 14: 559-67. doi: 10.1038/nrc3770

76. Ylösmäki E, Malorzo C, Capasso C, Honkasalo O, Fusciello M, Martins $B$, et al. Personalized cancer vaccine platform for clinically relevant oncolytic enveloped viruses. Mol Ther 2018; 26: 2315-25. doi: 10.1016/j. ymthe.2018.06.008

77. Shemesh CS, Hsu JC, Hosseini I, Shen BQ, Rotte A, Twomey P, et al Personalized cancer vaccines: Clinical landscape, challenges, and opportunities. Mol Ther 2021; 29: 555-70. doi: 10.1016/j.ymthe.2020.09.038

78. Ries S, Korn WM. ONYX-015: mechanisms of action and clinical potential of a replication-selective adenovirus. Br J Cancer 2002; 86: 5-11. doi: 10.1038/ sj.bjc.6600006

79. Liang M. Oncorine, the world first oncolytic virus medicine and its update in China. Curr Cancer Drug Targets 2018; 18: 171-6. doi: 10.2174/1568009 618666171129221503

80. Zheng $\mathrm{M}$, Huang J, Tong $\mathrm{A}$, Yang $\mathrm{H}$. Oncolytic viruses for cancer therapy: barriers and recent advances. Mol Ther - Oncolytics 2019; 15: 234-47. do 10.1016/j.omto.2019.10.007

81. Shen Y, Nemunaitis J. Herpes simplex virus 1 (HSV-1) for cancer treatment Cancer Gene Ther 2006; 13: 975-92. doi: 10.1038/sj.cgt.7700946

82. Andtbacka RHI, Kaufman HL, Collichio F, Amatruda T, Senzer N, Chesney $\mathrm{J}$, et al. Talimogene laherparepvec improves durable response rate in patients with advanced melanoma. J Clin Oncol 2015; 33: 2780-8. doi: $10.1200 / J C O .2014 .58 .3377$

83. Ramelyte E, Tastanova A, Balázs Z, Ignatova D, Turko P, Menzel U, et al. Oncolytic virotherapy-mediated anti-tumor response: a single-cell perspective. Cancer Cell 2021; 39: 394-406.e4. doi: 10.1016/j.ccell.2020.12.022

84. Kaufman HL, Maciorowski D. Advancing oncolytic virus therapy by understanding the biology. Nat Rev Clin Oncol 2021; 18: 197-8. doi: 10.1038/ s41571-021-00490-4

85. Haitz K, Khosravi H, Lin JY, Menge T, Nambudiri VE. Review of talimogene laherparepvec: a first-in-class oncolytic viral treatment of advanced melanoma. J Am Acad Dermatol 2020; 83: 189-96. doi: 10.1016/j. jaad.2020.01.039

86. Ribas A, Dummer R, Puzanov I, VanderWalde A, Andtbacka RHI, Michielin $O$, et al. Oncolytic virotherapy promotes intratumoral $T$ cell infiltration and improves anti-PD-1 immunotherapy. Cell 2017; 170: 1109-1119.e10. doi: 10.1016/j.cell.2017.08.027
87. Jahan N, Ghouse SM, Martuza RL, Rabkin SD. In situ cancer vaccination and immunovirotherapy using oncolytic HSV. Viruses 2021; 13: 1-27. doi: $10.3390 / \mathrm{v} 13091740$

88. Todo T, Martuza RL, Rabkin SD, Johnson PA. Oncolytic herpes simplex virus vector with enhanced MHC class I presentation and tumor cell killing. Proc Natl Acad Sci U S A 2001; 98: 6396-401. doi: 10.1073/pnas.101136398

89. Parato KA, Breitbach CJ, Le Boeuf F, Wang J, Storbeck C, llkow C, et al. The oncolytic poxvirus JX-594 selectively replicates in and destroys cancer cells driven by genetic pathways commonly activated in cancers. Mol Ther 2012 20: 749-58 doi: $10.1038 / \mathrm{mt} .2011 .276$

90. Guo ZS, Lu B, Guo Z, Giehl E, Feist M, Dai E, et al. Vaccinia virus-mediated cancer immunotherapy: Cancer vaccines and oncolytics. I Immunother Cancer 2019; 7: 1-21. doi: 10.1186/s40425-018-0495-7

91. Heo J, Reid T, Ruo L, Breitbach CJ, Rose S, Bloomston M, et al. Randomized dose-finding clinical trial of oncolytic immunotherapeutic vaccinia JX-594 in liver cancer. Nat Med 2013; 19: 329-36. doi: 10.1038/nm.3089

92. Foerster F, Galle PR. The current landscape of clinical trials for systemic treatment of HCC. Cancers 2021; 13: 1962. doi: 10.3390/cancers13081962

93. Gregg JR, Thompson TC. Considering the potential for gene-based therapy in prostate cancer. Nat Rev Urol 2021; 18: 170-84. doi: 10.1038/s41585021-00431-x

94. Gulley JL, Borre M, Vogelzang NJ, Ng S, Agarwal N, Parker CC, et al. Phase III Trial of PROSTVAC in asymptomatic or minimally symptomatic metastatic castration-resistant prostate cancer. J Clin Oncol 2019; 37: 1051-61. doi: 10.1200/JCO.18.02031

95. Madan RA, Arlen PM, Mohebtash M, Hodge JW, Gulley JL. Prostvac-VF: a vector-based vaccine targeting PSA in prostate cancer. Expert Opin Investig Drugs 2009; 18: 1001-11. doi: 10.1517/13543780902997928

96. Shi $T$, Song $X$, Wang $Y$, Liu F, Wei J. Combining oncolytic viruses with cance immunotherapy: establishing a new generation of cancer treatment. Front Immunol 2020; 11: 1-13. doi: 10.3389/fimmu.2020.00683

97. Moleirinho MG, Silva RJS, Alves PM, Carrondo MJT, Peixoto C. Current challenges in biotherapeutic particles manufacturing. Expert Opin Biol Ther 2019; 20: 451-65. doi: 10.1080/14712598.2020.1693541

98. Ungerechts G, Bossow S, Leuchs B, Holm PS, Rommelaere J, Coffey M, et al. Moving oncolytic viruses into the clinic: clinical-grade production, purification, and characterization of diverse oncolytic viruses. Mol Ther - Methods Clin Dev 2016; 3: 16018. doi: 10.1038/mtm.2016.18

99. Ghosh S, Brown AM, Jenkins C, Campbell K. Viral vector systems for gene therapy: a comprehensive literature review of progress and biosafety challenges. Appl Biosaf 2020; 25: 7-18. doi: 10.1177/1535676019899502

100. Merten OW, Schweizer M, Chahal P, Kamen AA. Manufacturing of viral vectors for gene therapy: part I. Upstream processing. Pharm Bioprocess 2014; 2: 183-203. doi: 10.4155/pbp.14.16

101. van der Loo JCM, Wright JF. Progress and challenges in viral vector manufacturing. Hum Mol Genet 2016; 25: R42-52. doi: 10.1093/hmg/ddv451

102. Ferreira MV, Cabral ET, Coroadinha AS. Progress and perspectives in the development of lentiviral vector producer cells. Biotechnol J 2021; 16. doi: 10.1002/biot.202000017

103. Tomás HA, Rodrigues AF, Carrondo MJT, Coroadinha AS. LentiPro26: novel stable cell lines for constitutive lentiviral vector production. Sci Rep 2018, 8: 1-11. doi: 10.1038/s41598-018-23593-y

104. Felberbaum RS. The baculovirus expression vector system: a commercial manufacturing platform for viral vaccines and gene therapy vectors. Biotechnol J 2015; 10: 702-14. doi: 10.1002/biot 201400438

105. Kurasawa JH, Park A, Sowers CR, Halpin RA, Tovchigrechko A, Dobson CL, et al. Chemically defined, high-density insect cell-based expression system for scalable AAV vector production. Mol Ther Methods Clin Dev 2020; 19: 330-40. doi: 10.1016/j.omtm.2020.09.018

106. Gupta P, Monge M, Boulais A, Chopra N, Hutchinson N. Single-use process platforms for responsive and cost-effective manufacturing. In: Eibl R, Eibl D, editors. Single-use technology in biopharmaceutical manufacture. Hoboken, NY, USA: John Wiley \& Sons, Inc 2019. p. 201-10. doi: 10.1002/9781119477891.ch16 
107. Minh A, Kamen AA. Critical assessment of purification and analytical technologies for enveloped viral vector and vaccine processing and their current limitations in resolving co-expressed extracellular vesicles. Vaccines 2021; 9: 823. doi: 10.3390/vaccines9080823

108. Merten O-W, Schweizer M, Chahal P, Kamen A. Manufacturing of viral vectors: part II. Downstream processing and safety aspects. Pharm Bioprocess 2014; 2: 237-51. http://www.future-science.com/doi/abs/10.4155/ pbp.14.15\%0Apapers2://publication/doi/10.4155/pbp.14.15

109. Kaemmerer WF. How will the field of gene therapy survive its success? Bioeng Transl Med 2018; 3: 166-77. doi: 10.1002/btm2.10090

110. Salzman R, Cook F, Hunt T, Malech HL, Reilly P, Foss-Campbell B, et al. Addressing the value of gene therapy and enhancing patient access to transformative treatments. Mol Ther 2018; 26: 2717-26. doi: 10.1016/j. ymthe.2018.10.017

111. Capra E, Godfrey A, Loche A, Smith J. Innovation in viral-vector gene therapy: unlocking the promise. [cited 2021 Dec 24]. Available at https:// www.mckinsey.com/industries/life-sciences/our-insights/gene-therapyinnovation-unlocking-the-promise-of-viral-vectors

112. Wolf MW, Reichl U. Downstream processing of cell culture-derived virus particles. Expert Rev Vaccines 2011; 10: 1451-75. doi: 10.1586/erv.11.111

113. Martin NT, Bell JC. Oncolytic virus combination therapy: killing one bird with two stones. Mol Ther 2018; 26: 1414-22. doi: 10.1016/j. ymthe.2018.04.001

114. Bridle BW, Boudreau JE, Lichty BD, Brunellière J, Stephenson K, Koshy S, et al. Vesicular stomatitis virus as a novel cancer vaccine vector to prime antitumor immunity amenable to rapid boosting with adenovirus. $\mathrm{Mol}$ Ther 2009; 17: 1814-21. doi: 10.1038/mt.2009.154 